\title{
Altersgemischter Unterricht in kleinen Schulen im ländlichen Raum
}

\section{Andrea Raggl}

Im Rahmen dieses Beitrags werden die Besonderheiten kleiner Schulen und die Praxis des altersgemischten Unterrichts in kleinen Schulen im ländlich-alpinen Raum beschrieben. Der altersgemischte Unterricht stellt im ländlichen Raum vor allem eine notwendige organisatorische Massnahme dar, um kleine Schulen mit geringen SchülerInnenzahlen erhalten zu können. Die im Forschungsprojekt "Schulen im alpinen Raum" beteiligten SchulleiterInnen und LehrerInnen erkennen jedoch auch die pädagogischen Chancen der kleinen Organisationsform und des altersgemischten Unterrichts. Einige nützen den zur Verfügung stehenden Gestaltungsspielraum für die Entwicklung innovativer didaktischer Modelle. Es zeigte sich, dass die Umsetzung altersgemischten Unterrichts Lehrpersonen insgesamt vor grosse Herausforderungen stellt, insbesondere jene in sehr kleinen "Ein-LehrerInnen-Schulen» (vgl. Raggl2011).

Im ländlich-alpinen Raum gibt es aufgrund der geographischen Lage viele kleine und zum Teil sehr kleine Schulen. Im Rahmen des Interreg-Projekts «Schule im alpinen Raum» wurde die Situation kleiner Schulen in einer grenzüberschreitenden Zusammenarbeit der Pädagogischen Hochschulen Vorarlberg, Graubünden, St. Gallen und Wallis untersucht (Müller, Keller, Kerle, Raggl \& Steiner, 2011). In vielen kleinen Schulen wird der Unterricht altersgemischt organisiert, weil die SchülerInnenzahl nicht ausreicht, um für jede Jahrgangsstufe eine altershomogene Lerngruppe zu bilden. Der altersgemischte Unterricht stellt hier vor allem eine organisatorische Notwendigkeit dar, um kleine Schulen erhalten zu können. Im Rahmen dieses Beitrags werden die Besonderheiten kleiner Schulen beschrieben und die Praxis des altersgemischten Unterrichts analysiert.

\section{Forschungsstand}

Im deutschsprachigen Raum gibt es bislang wenige empirische Arbeiten über kleine Schulen im ländlichen Raum. Es gibt einige AutorInnen, die sich allgemein 
mit der Situation kleiner Schulen auseinandersetzen (Fickermann, Weishaupt \& Zedler, 1998; Rückriem, 1965; Sandfuchs, Stange \& Kost, 1997). Der Blick auf die internationale Forschungslandschaft zeigt, dass "(small) rural schools» im anglo-amerikanischen Raum (Arnold, Newman, Gaddy \& Dean, 2005; Hargreaves, Kvalsund \& Galton, 2009), aber auch in Skandinavien (Kalaoja \& Pietarinen, 2009) und Australien (Starr \& White, 2008) mehr Beachtung in der aktuellen Schulforschung finden. Es zeigen sich in diesen Studien ähnliche Ausgangssituationen und Problemlagen kleiner Schulen: die grosse Bedeutung der kleinen Schule für die ländliche Region, Rückgang an SchülerInnen, Angst vor Schliessung, etc. In den Studien werden kleine Schulen sehr unterschiedlich definiert, in vielen Fällen ist bereits eine Schule mit weniger als 100 SchülerInnen eine «kleine» Schule. Im Rahmen des oben erwähnten Forschungsprojekts wurden jene Schulen als "kleine Schulen» bezeichnet, welche aufgrund ihrer geringen Schülerzahlen altersgemischt organisiert sind (Raggl, 2011).

Einen Forschungsüberblick über altersgemischten Unterricht geben Rossbach (2003) und Hanke (2007) für den deutschsprachigen Raum, für den amerikanischen Raum Gutiérrez und Slavin (1992). Sehr praxisnahe Beiträge finden sich in Laging (2003), De Boer, Burk und Heinzel (2007) sowie Hahn und Berthold (2010). In den letzten Jahren entstanden im deutschsprachigen Raum - vor allem durch die Neukonzeption der Schuleingangsphase - einige empirische Studien zum Lernen in altersgemischten Lerngruppen (Berthold, 2008; Kucharz \& Wagener, 2009). Trachsler (2008) weist darauf hin, dass in den Schulversuchen der letzten Jahren jedoch selten auf die langjährigen Erfahrungen von kleinen Schulen im Zusammenhang mit altersgemischten Unterricht zurückgegriffen wurde (S. 5). Insgesamt lässt sich beobachten, dass die Praxis des altersgemischten Unterrichts in kleinen Schulen selten in den Fokus empirischer Studien rückt, obwohl es im ländlichen Raum sehr viele kleine Schulen gibt. Poglia und Strittmatter (1983) forderten bereits vor 30 Jahren in ihrem Situationsbericht mehr Aufmerksamkeit für «Mehrklassenschulen» von Seiten der Bildungspolitik. Dies ist vor allem auch deshalb notwendig, weil die Zahl kleiner Schulen im ländlichen Raum aufgrund der Entwicklung der SchülerInnenzahlen weiter zunimmt.

Aufgrund der dünnen Forschungslage hatte die Studie einen explorativen Charakter und die Forschungsfragen waren eher breit angelegt. In diesem Beitrag stehen folgende Fragen im Zentrum: Wie sieht die Arbeitssituation für SchulleiterInnen und Lehrpersonen in den beteiligten kleinen Schulen aus? Wie wird der altersgemischte Unterricht in diesen Schulen umgesetzt? Welche Chancen und Herausforderungen erleben die Lehrpersonen bei der Umsetzung? 


\section{Methodisches Vorgehen}

Im Rahmen des Interreg Projekts «Schule im alpinen Raum» wurde die Praxis des altersgemischten Unterrichts in acht kleinen Schulen in Vorarlberg sowie jeweils zwei Schulen in Graubünden, St. Gallen und im Wallis untersucht (Tab. 1).

Tabelle 1: Kleine Schulen (Schuljahr 2009/10)

\begin{tabular}{lcc}
\hline & $\begin{array}{c}\text { Anzahl } \\
\text { SchülerInnen }\end{array}$ & Schulstufen \\
\hline Schulen Vorarlberg & 11 & \\
Volksschule V1 & 11 & $1 .-4$. \\
Volksschule V2 & 12 & $1 .-4$. \\
Volksschule V3 & 10 & $1 .-4$. \\
Volksschule V4 & 33 & $1 .-4 ., 5 .-8$. \\
Volksschule V5 & 22 & $1 .+2 ., 3 .+4$. \\
Volksschule V6 & 52 & $1 .+2 ., 3 .+4$. \\
Volksschule V7 & 50 & $1 .+2 ., 3 .+4$. \\
Volksschule V8 & & $1 .+4 ., 2 ., 3$. \\
Schulen Graubünden, St. Gallen und Wallis & 10 & $1 .-6$. \\
Primarschule G1 & 22 & $1 .-3 ., 4 .-6$. \\
Primarschule G2 & 26 & $1 .-3 ., 4 .-6$. \\
Primarschule SG1 & 30 & $1 .-3 ., 4 .-6$. \\
Primarschule SG2 & 33 & $1.3 ., 4 .-6$. \\
Primarschule W1 & 33 & $1 .+2 ., 3 .+4 ., 5 .+6$. \\
Primarschule W2 & &
\end{tabular}

Aus Tabelle 1 geht hervor, dass 5 der beteiligten Schulen sehr kleine Schulen mit bis zu 12 SchülerInnen sind, die in Vorarlberg von der 1. bis zur 4. Schulstufe und in der Schweiz von der 1. bis zur 6. Schulstufe altersgemischt von einer Klassenlehrperson unterrichtet werden. In Vorarlberg werden diese Schulen als «Kleinstschulen» bezeichnet, in einigen Kantonen der Schweiz als "Gesamtschulen». Aus der Tabelle geht weiters hervor, dass zumeist die aufeinander folgenden Klassen gemeinsam unterrichtet werden. Nur Schule V8 zeigt eine andere Kombination: Hier wurde ein sehr geburtenschwacher Jahrgang mit nur vier SchülerInnen gemeinsam mit der ersten Klasse zusammengelegt, da sich diese Kombination schulorganisatorisch als leichter umsetzbar erwies. Eine der beteiligten sehr kleinen Schulen (V4) wurde ein Jahr nach der Datenerhebung geschlossen, da die Schülerzahl im Schuljahr 2010/11 auf fünf SchülerInnen sank. Es war die letzte kleine Schule in Vorarlberg mit einer Oberstufe. 
Die Datenerhebung erfolgte mit qualitativen und quantitativen Methoden. Es wurden in den 14 Schulen Leitfaden-Interviews mit 14 SchulleiterInnen und 10 Lehrpersonen durchgeführt sowie mit 3 Schulaufsichtspersonen in Vorarlberg. Die Interviews wurden transkribiert, die Inhaltsanalyse erfolgte mit Hilfe des Softwareprogramms Atlas.ti. Für die Fragebogenerhebung wurde vom Projektteam ein gemeinsamer Fragebogen entwickelt, der neben anderen Aspekten, auch einige Fragen zum altersgemischten Unterricht enthielt und in insgesamt 44 Schulen zum Einsatz kam (110 Lehrpersonen, 635 SchülerInnen, 780 Eltern). Teilnehmende Beobachtung ermöglichte vertiefende Einblicke in die Praxis des altersgemischten Unterrichts.

\section{Ergebnisse}

Im Folgenden werden zunächst einige Besonderheiten kleiner Schulen beschrieben und anschliessend die zentralen Ergebnisse zum altersgemischten Lernen angeführt.

\section{Besonderheiten kleiner Schulen}

Kleine Schulen im ländlichen Raum weisen einige Besonderheiten auf, die sie von grösseren Schulen unterscheiden. Eine organisatorische Besonderheit kleiner Schulen stellt die Rolle der Schulleitung dar, da diese in kleinen Schulen neben der Unterrichtstätigkeit ausgeübt werden muss. Aus dem Datenmaterial geht hervor, dass viele SchulleiterInnen kleiner Schulen sich vor allem als LehrerInnen sehen:

Also ich empfinde mich selbst zu $95 \%$ als Lehrerin, das muss ich schon sagen und die Leiterinnentätigkeit, ja, das läuft eben nebenbei her. (Schulleiterin V1)

Aufgrund dieses ausgeprägten Selbstverständnisses als LehrerInnen wird in diesem Beitrag in weiterer Folge von "LehrerInnen" gesprochen, auch wenn es sich um SchulleiterInnen handelt.

Kleine Schulen zeigen sich immer wieder als innovative Schulen (Waugh, 1990; Vulliamy \& Webb, 1995). Einige der Lehrpersonen weisen darauf hin, dass sich die kleine Organisationsform für die Umsetzung neuer Ideen besonders eignet:

Die Riesenvorteile, die so eine kleine Schule hat. (...) Der Name sagt schon Kleinschule, das ist überschaubar, ich kann Ideen sofort umsetzen. Ich bin sehr nahe an den Kindern, ich bin sehr nahe an Eltern (...) Da kann man gut miteinander kooperieren, Ideen umsetzen, gemeinsam Schule entwickeln. (Schulleiter V6) 
Auf die Frage "Was ist das Besondere einer kleinen Schule?», heben einige der interviewten Lehrpersonen sowie VertreterInnen des Schulmanagements die «familiären Beziehungen» zwischen SchülerInnen und Lehrpersonen als sehr positiv hervor und beschreiben die kleine Schule als «Familienbetrieb»:

Es ist ein Familienbetrieb (...) vergleichbar mit einer Grossfamilie (...) und da findet ein Schultag in dieser familiären Atmosphäre statt. (Landesschulinspektor Vorarlberg)

Durch die Betonung des Familiencharakters von kleinen Schulen wird die Nähe zwischen dem Lehren und Lernen in kleinen Schulen und reformpädagogischen Bewegungen (Oelkers, 1989) deutlich. Der familiäre Charakter wird durch die geringe Schülerzahl verstärkt. Die in familienähnlichen Konstellationen vorzufindende grosse Nähe zwischen Lernenden und der Lehrperson kann sich auf das Lernen der Kinder positiv auswirken, sie kann jedoch auch hinderlich sein, wenn sich die Beziehungen schwierig gestalten. Die grosse Abhängigkeit von einer Lehrperson, welche die Lernerfahrungen der SchülerInnen in einem besonderen Mass prägt, wurde von einem Schulleiter treffenderweise als «Schicksalsgemeinschaft» bezeichnet:

Das ist natürlich eine Schicksalsgemeinschaft auf Gedeih und Verderb, (...) wie es eben menschlich ist, wenn man mit einem Schüler nicht so gut kann oder er mit einem nicht, das dauert dann (...) Jahre. (Schulleiter V4)

Diese «menschliche» Seite kann in kleinen Schulen grosse Auswirkungen auf die (Schul)Laufbahn einzelner Kinder haben. Sandfuchs (1997) sieht in der Problematik, dass «kein Schüler ihm entweichen kann» (S. 17), eine zentrale Problematik sehr kleiner Schulen im ländlichen Raum. Trachsler (2008) weist darauf hin, dass die langjährige pädagogische Beziehung gleichzeitig einen entscheidenden «Qualitäts- als auch Risikofaktor» (S. 49) darstellt. Dies geht auch aus den folgenden Zeilen einer Mutter hervor, welche in einer offenen Frage im Elternfragebogen anführt:

Wir haben derzeit das Glück, dass unsere Schule von einer ausgezeichneten Lehrerin geführt wird. Wir hatten schon das Gegenteil, was für Kinder in altersgemischten Klassen ein besonderes Problem darstellt. (Mutter V3)

Hier wird es als «Glück» wahrgenommen und nicht als Selbstverständlichkeit, dass die Schule von einer «ausgezeichneten Lehrerin geführt wird». Für Kinder und Eltern aber auch für die Lehrperson kann es eine äusserst belastende Situation darstellen, wenn sich Beziehungen schwierig gestalten. Probleme können sich in kleinen Schulen im ländlichen Raum durch die isolierte Situation verschärfter zeigen als in grösseren Schulen. Hier stehen besonders sehr kleine "Ein-LehrerInnen-Schulen» vor grossen Herausforderungen. Ein Vertreter des regionalen Schulmanagements in Vorarlberg findet für die dort anzufindende Einsamkeit von LehrerInnen sehr klare Worte: 
Weil das Problem an Kleinschulen ja ist: Ich habe niemanden, mit dem ich drüber reden kann. Ich vereinsame in den Bergen! (Bezirksschulinspektor V)

Die einsame Tätigkeit wird dadurch verschärft, dass gerade in kleinen Gemeinden oft eine hohe Erwartungshaltung gegenüber Lehrpersonen besteht, sich für das Dorfleben zu engagieren. Die Nähe zur Dorfbevölkerung steht in einem Spannungsfeld mit der professionellen Tätigkeit und wird von Lehrpersonen als "Gratwanderung» beschrieben. Aus dem Datenmaterial geht hervor, dass sich Lehrpersonen nicht mehr so stark als «Kulturträger» - im Sinne des meist männlichen Dorfschullehrers früherer Zeiten - sehen, sondern die professionelle Ebene ihrer Tätigkeit betonen. Sie distanzieren sich zum Teil sehr bewusst von der allumfassenden Vereinnahmung ihrer Person.

\section{Der altersgemischte Unterricht in kleinen Schulen}

\section{Notwendigkeit und Chance}

In vielen kleinen Schulen wird aufgrund der geringen Schülerzahl altersgemischt unterrichtet. In Vorarlberg wurden im Schuljahr 2009/10 40\% der Grundschulen altersgemischt geführt (Amt der Vorarlberger Landesregierung, 2011, S. 7), in Graubünden 34\% der Schulklassen auf der Primarstufe, im deutschsprachigen Wallis über 40\% und in St. Gallen 23\%. Der altersgemischte Unterricht kann für einige Stunden pro Woche oder auf einzelne Fächer begrenzt sein, aber auch die gesamte Unterrichtszeit umfassen. Für diese Organisationsform werden unterschiedliche Begriffe synonym verwendet: jahrgangsübergreifend, jahrgangsgemischt, altersheterogen, altersgemischt oder jahrgangskombiniert (Kucharz, 2009, S. 339). In der Schweiz ist auch der Begriff «altersdurchmischt» üblich.

In ländlichen Regionen in Vorarlberg und den beteiligten Kantonen gibt es eine lange Tradition altersgemischten Unterrichts. Er stellt hier zum Teil eine unhinterfragte Selbstverständlichkeit sowohl seitens der Lehrpersonen als auch der Eltern dar:

Hier ist es einfach so, es ist vorgegeben und das wissen die Eltern schon von Beginn weg. Sie können nicht wählen, die Schule hat nicht das Modell umgestellt oder so, das ist einfach so. (Lehrerin G1)

Sie sind es gewohnt, sie sind selber schon in diese Unterrichtsform gegangen, für sie ist es Alltag und normal. (Schulleiterin V4)

Viele der Eltern haben selbst eine kleine Schule mit altersgemischtem Unterricht besucht und auch viele der Lehrpersonen (58\%), wie aus der schriftlichen Befragung hervorgeht. Einige der Lehrpersonen haben ihr gesamtes LehrerInnenleben in einer kleinen Schule mit altersgemischten Klassen unterrichtet. Für sie stellt diese Unterrichtsform etwas sehr Vertrautes, manchmal allzu Vertrautes dar. 
Die pragmatische Begründung, altersgemischte Lerngruppen nur bzw. vor allem deshalb einzurichten, um Schulen mit geringer Schülerzahl aufrechtzuerhalten, unterscheidet sich von einer pädagogischen Begründung grösserer (Reform-)Schulen, die sich bewusst für die Bildung altersgemischter Lerngruppen entscheiden (Hanke, 2007). Breuer und Simon (2001) sprechen in diesem Zusammenhang von einem "schulorganisatorischen Notnagel»: "Jahrgangsübergreifender Unterricht galt in der Vergangenheit bei manchen Lehrkräften, insbesondere aber bei manchen Eltern und in der Öffentlichkeit häufig als schulorganisatorischer Notnagel, wenn nicht genügend SchülerInnen vorhanden waren.» (S. 32) Auch ein Schulleiter einer kleinen Schule in Vorarlberg äussert sich kritisch über die oft vorzufindende Praxis des altersgemischten Unterrichts in kleinen Schulen. Im Titel eines Artikels spricht der Reformpädagoge einen zentralen Aspekt an: "Jahrgangsmischung an einer Kleinschule - Über das Müssen und das Wollen der Altersmischung»:

Altersmischung an Kleinschulen gab es immer schon. Methodisch wurde und wird dieser Gegebenheit meist mittels Abteilungsunterricht begegnet. Die Lehrperson ist also GestalterIn des Unterrichts bzw. der Arbeit in den verschiedenen Abteilungen. Wenn die Durchmischung aber nicht als Manko oder als eben gegeben hingenommen wird, sondern sogar erwünscht ist, dann könnte die Organisation an vielen Kleinschulen völlig anders aussehen (Grabher, 2002, S. 511).

Trachsler (2008) weist darauf hin, dass «die zur Zeit gängige Unterscheidung zwischen ,organisatorisch bedingten Mehrjahrgangsklassen ' und ,auf pädagogischen Überzeugungen gründenden Lehr-Lern-Formen mit gemischten Jahrgängen" " (S. 4) wenig stichhaltig scheint. Aus dem Datenmaterial geht hervor, dass in vielen kleinen Schulen sowohl pragmatische als auch pädagogische Begründungen anzutreffen sind.

\section{Pädagogische Chancen des altersgemischten Unterrichts}

Als wesentliche pädagogische Chance des altersgemischten Unterrichts sehen Lehrpersonen kleiner Schulen vor allem das gegenseitige Helfen der Kinder. Lehrpersonen erleben die SchülerInnen altersgemischter Lerngruppen als besonders selbständig. Ein weiterer Vorteil wird darin gesehen, dass SchülerInnen in einer altersgemischten Lerngruppe mehr Entwicklungszeit zugestanden und das Aufrücken in die nächste Schulstufe individueller gestaltet werden kann:

Als einen grossen Vorteil sehe ich, dass es einfach nicht auffällt, wenn ein Kind zum Beispiel ein Jahr wiederholt, wenn es ein Vorschulkind ist (...) das fällt so im Klassenverband überhaupt nicht auf (...) dass ihm das als Vorschuljahr angerechnet wird, (...) weil es bleibt ja irgendwo in der Gruppe und das ist schon ein Vorteil. (Schulleiterin V3) 
Der altersgemischte Unterricht «entdramatisiert» Entwicklungsunterschiede zwischen Kindern (Heinzel, 2007, S. 38), dadurch wird die Schuleingangsphase entlastet (Berthold, 2008). Auch das Problem der Klassenwiederholung kann im altersgemischten Unterricht deutlich entschärft werden, da Kinder mit besonderen Förderbedürfnissen in ihrer vertrauten Lerngruppe bleiben können. Der altersgemischte Unterricht sichert damit die soziale Kontinuität innerhalb der Lerngruppe (Kucharz \& Wagener, 2009, S. 11). Neu hinzukommende SchülerInnen kommen in eine Gruppe, in der die Regeln und Rituale bereits bestehen und können sich an älteren SchülerInnen orientieren. Lehrpersonen beobachten, dass Kinder sehr schnell von anderen Kindern lernen:

Da hat es natürlich grosse Vorteile, als wenn (...) da 20 oder 25 Erstklässler herkommen und von $\mathrm{A}$ bis $\mathrm{Z}$ muss ich ihnen als Lehrperson alles erklären. Und ich habe auf der anderen Seite Zweit- und Drittklässler, die wissen wie der Hase läuft, da kann ich eine Paarbildung machen, ein Helfersystem. Da bin ich viel schneller und viel effizienter, weil das Kind lernt vom Kind viel schneller als von mir. (Schulleiter V4)

Lehrpersonen sehen die Vorteile des «Helfersystems» vor allem darin, dass sich SchülerInnen untereinander besser verständigen können:

Wenn der ältere (...) Schüler mit dem anderen zusammenarbeitet, (...) er erklärt die Sache vielleicht manchmal sogar besser als der Lehrer, weil er auf der gleichen Stufe ist. (Schulleiterin W1)

In der gemeinsamen Reflexion mit anderen Kindern liegt eine wichtige Möglichkeit «für die Ausdifferenzierung bzw. Transformation ihrer Erklärungsmodelle» (Hahn \& Berthold, 2010, S. 9). Hilfe und Unterstützung durch (ältere) MitschülerInnen ist jedoch nicht immer erwünscht. Ob die Hilfe angenommen werden kann, hängt auch davon ab, von wem sie angeboten wird. Kinder finden schnell heraus, wer ihnen am besten helfen kann bzw. von wem sie sich am liebsten helfen lassen. Hilfesituationen im Unterricht beruhen auf einem Kompetenzgefälle zwischen Hilfegeber und Hilfenehmer. Der belehrende Habitus gegenüber jüngeren SchülerInnen kann sich negativ auf die Interaktion zwischen den SchülerInnen auswirken (Krappmann \& Oswald, 1995). Lehrpersonen weisen darauf hin, dass es für die älteren SchülerInnen manchmal zu viel sein kann, immer in die Rolle der verantwortungsvollen «Grossen» gedrängt zu werden:

Und natürlich auch das Rücksicht-nehmen-Müssen, irgendwo ist es schön, wenn sie es lernen. Auf der anderen Seite, Viertklässler untereinander sind einfach mal wilder als die Erstklässler. Schauen müssen, dass die Kleinen nicht untergehen, permanent irgendwo doch ein bisschen so die Rolle der Grossen haben und das als Zehnjähriger schon, das sehe ich nicht unbedingt immer so als Vorteil. (Schulleiterin V3) 
Aus der schriftlichen Befragung der SchülerInnen geht hervor, dass so gut wie alle SchülerInnen (90\%) gern helfen und der Grossteil (79\%) davon überzeugt ist, dass sie ihren MitschülerInnen Dinge gut erklären können. Die Aussage «Das Arbeiten mit älteren und jüngeren SchülerInnen macht mir Spass», stimmt für $37 \%$ der SchülerInnen «genau» und für 44\% «eher». Die Kompetenz, sinnvoll zu helfen, kann jedoch nicht vorausgesetzt werden (Wagener, 2007, S. 132). Lehrpersonen erleben, dass das Helfen auch «gelernt werden muss» (Schulleiterin V2).

\section{Heterogenitätsbewusstsein von Lehrpersonen kleiner Schulen}

Der Differenzierungsanspruch ist in jeder Lerngruppe ein wichtiger Bestandteil pädagogischer Bemühungen, in einer altersgemischten Lerngruppe verstärkt sich die Notwendigkeit zu differenzieren (Hagstedt, 2003, S. 34). Die Altersunterschiede der SchülerInnen können das Bewusstsein für die Heterogenität der Lerngruppe verstärken:

Der grösste Vorteil ist sicher, dass man den Schülern gerecht wird. Das heisst, das Individualisierende, Differenzierende ist das, was man tagtäglich tun muss. (...) Aber ich kann mir vorstellen, wenn ich auch in einer jahrgangshomogenen Klasse wäre, dass es einfach unterschiedliche Niveaus gibt, dass man da auch individualisierend arbeiten sollte oder müsste. (Schulleiter V6)

Ich meine, es weiss jeder, dass du auch differenzieren musst, wenn du eine Stufe hast, aber es ist eben anders noch einmal klarer. (...) Es wird so gedacht, dass es eine homogene Gruppe ist - ist es nicht - aber in der anderen Klasse weisst du, dass es das nicht ist. (Landesschulinspektorin V)

Hier wird deutlich, dass altersgemischte Lerngruppen es erschweren, an der «Fiktion der Homogenität» (Tillmann, 2004) festzuhalten. In der schriftlichen Befragung von Lehrpersonen zeigt sich, dass einige die altersgemischte Lerngruppe als grosse Herausforderung erleben: Bei der Aussage «Ich fühle mich durch die grosse Heterogenität der SchülerInnen überfordert", geben $47 \%$ an «trifft eher zu» und 14\% «trifft zu». In den Interviews zeigt sich zum Teil der Wunsch nach einer (alters)homogeneren Lerngruppe:

Ich persönlich freue mich auf das Jahr, wo ich in eine Klasse hineinlaufe, wo alle gleichaltrig sind, weil ich mir es von der Vorbereitung her einfach viel einfacher und weniger zeitintensiv vorstelle. (Schulleiterin V3)

Aus der schriftlichen Befragung geht weiters hervor, dass Lehrpersonen gern mit altersgemischten Lerngruppen arbeiten, welche aus zwei Jahrgängen bestehen, dass mehr als drei Jahrgänge aber als grosse Herausforderung erlebt werden. Lehrpersonen altersgemischter Klassen erleben vor allem den hohen Vorbereitungsaufwand als grosse Herausforderung. So geben 38\% der Lehrpersonen an es «trifft zu», dass sie den Vorbereitungsaufwand als sehr hoch erleben, und 
$51 \%$, es «trifft eher zu». Der Vorbereitungsaufwand wird besonders von Lehrpersonen von "Ein-LehrerInnen-Schulen» mit vier bzw. sechs Schulstufen als sehr hoch eingestuft. Vor allem JunglehrerInnen, die in den ersten Jahren ihrer Tätigkeit in einer sehr kleinen Schule arbeiten, erleben den Arbeitsaufwand als sehr belastend. Einzelne Lehrpersonen weisen darauf hin, dass der Zeitaufwand für die Vorbereitung des altersgemischten Unterrichts zwar höher ist, dass sich jedoch der Zeitaufwand für die Korrekturarbeit aufgrund der meist geringen Schülerzahl in kleinen Schulen deutlich verringert.

\section{Gestaltung des altersgemischten Unterrichts in kleinen Schulen}

In den meisten der 14 kleinen Schulen stellt der «traditionelle» Abteilungsunterricht die zentrale Organisationsform dar. Bei der schriftlichen Befragung geben $26 \%$ der Lehrpersonen an, dass es für sie zutrifft, dass sie den altersgemischten Unterricht vor allem in Form von Abteilungsunterricht umsetzen, für 54\% trifft dies «eher zu». Ähnliche Ergebnisse zeigen sich in früheren Studien (Hanke, 2007; Poglia \& Strittmatter, 1983). Beim Abteilungsunterricht befinden sich zwar Kinder unterschiedlichen Alters in einem Raum, sie werden aber nach «Abteilungen», der Schulstufe der SchülerInnen entsprechend, getrennt unterrichtet. Während die Lehrperson mit einer altershomogenen Lerngruppe beschäftigt ist, arbeiten die anderen SchülerInnen in "Stillarbeit» an ihren Aufgaben. Poglia und Strittmatter (1983) bezeichnen diese Form als «Managementansatz», bei der der Unterricht in «Mini-Monoklassen» (S. 45) synchronisiert nebeneinander abläuft. In der Literatur wird der Abteilungsunterricht oft als veraltete bzw. überholte Unterrichtsmethode kritisiert (Peschel, 2007, S. 104). Aus den Interviews und Unterrichtsbeobachtungen geht hervor, dass der Abteilungsunterricht zwar häufig eingesetzt wird, jedoch immer wieder durch offenere und altersgemischt gestaltete Lernsituationen erweitert wird. In der schriftlichen Befragung trifft die Aussage «Ich setze altersgemischten Unterricht so um, dass Lernende auch mit SchülerInnen aus anderen Jahrgängen zusammenarbeiten können», für 36\% der Lehrpersonen «zu» und für 43\% «eher zu». Wie die Zusammenarbeit zwischen Lernenden unterschiedlicher Jahrgangsstufen erfolgt, ist in den Schulen sehr unterschiedlich. Kooperative Lernsituationen über die Schulstufen hinweg finden beispielsweise in Form von Stationenlernen oder Projekten statt. Lehrpersonen weisen darauf hin, dass die kleine Gruppengrösse es ermöglicht, die Interessen der SchülerInnen stärker in den Unterricht mit einzubeziehen:

$\mathrm{Du}$ kannst mehr interessensorientiert arbeiten, das ist auch ganz wichtig. Vielmal kommen Kinder mit Ideen und dadurch dass es so klein ist (...) können wir dann auch sagen, das interessiert vielleicht die anderen Schüler aus den anderen Schulstufen auch, dann machen wir einfach ein Projekt. (Schulleiter V6) 
Viele Lehrpersonen organisieren den altersgemischten Unterricht so, dass ein gemeinsames Thema die verschiedenen Altersstufen miteinander verbindet:

Zum Beispiel Mensch und Umwelt habe ich von der 4.-6. Klasse das gleiche Thema. Wir haben gewisse Lernziele, die wir miteinander erarbeiten und dann haben die 6. Klässler einfach mehr Lernziele. Sie haben das Ganze detaillierter, genauer und müssen mehr informiert sein. Die interessierten, guten 4. Klässler können mehr machen als sie müssten. Manchmal hat ein Viertklässler dieselben Lernziele wie der Sechstklässler. (Schulleiter SG2)

Nach einem gemeinsamen Einstieg arbeiten die SchülerInnen an verschiedenen Aufgaben, die sich in ihrem Umfang aber auch in ihrem Schwierigkeitsgrad unterscheiden, entsprechend einem Lernen am Gemeinsamen Gegenstand (Feuser, 2002). Einige der Lehrpersonen öffnen den altersgemischten Unterricht mit Hilfe von Wochen- bzw. Tagesplänen. Der Wochenplan schafft Lehrpersonen die notwendigen «Freiräume», um gezielt mit einer Kleingruppe arbeiten zu können:

Also ein wichtiger Teil ist für mich persönlich die Wochenplanarbeit. Bei mir bekommt jedes Kind am Montag einen eigenen Plan (...) und wie heute kann ich einmal zu den Dritt- und Viertklässlern sagen: «Wochenplan». Und der schafft mir dann wieder Freiraum. (...) Also das ist etwas Wichtiges und auch, dass sie gewöhnt sind, selbstständig zu arbeiten. (Schulleiterin V3)

Einige Lehrpersonen arbeiten mit individuellen Lernprogrammen:

Einen grossen Teil des Unterrichts machen die Kinder wirklich so ihr individuelles Programm (...) nach dem Stand, an dem sie stehen und nicht unbedingt der Klasse (...) weil zum Beispiel zweite, dritte, vierte Klasse hat je zwei Kinder drin, die aber total unterschiedlich sind. Also das ist so hauptsächlich, jedem Kind sein Programm in den Hauptfächer. (Lehrperson GR1)

Individuelle Lernprogramme orientieren sich am Leistungsstand der SchülerInnen und nicht an ihrem Alter bzw. der Schulstufe.

Eine innovative Variante der Umsetzung altersgemischten Unterrichts stellt der in einer Vorarlberger Schule (V7) praktizierte "Werkstattunterricht» dar. SchülerInnen von vier Schulstufen arbeiten zwei Tage in der Woche regelmässig für zwei Stunden zusammen. Dafür werden die ansonsten in 1./2. sowie 3./4. Schulstufe aufgeteilten Lerngruppen gemeinsam vom LehrerInnen-Team unterrichtet. Die Lehrpersonen bereiten den Werkstattunterricht gemeinsam vor. Ein grösserer Themenbereich wird in Form von "Ateliers»(Freinet-Pädagogik) zur selbständigen Bearbeitung angeboten. Die SchülerInnen erhalten eine Übersicht über die Stationen als Orientierungshilfe und regeln selbst, wann sie welche Station mit wem bearbeiten. Manche der Stationen sind für die 3./4. Schulstufe, andere für die 1./2. und einige werden bewusst für alle angeboten. Der Schulleiter verfügt über mehr als 25 Jahre Erfahrung mit altersgemischten 
Lerngruppen und der Werkstattunterricht stellt ein Entwicklungsprojekt dar. In den ersten Jahren seiner Lehrtätigkeit dominierte noch der von ihm als «klassisch» bezeichnete "Abteilungsunterricht». Der Schulleiter erklärt, dass dies in der Ausbildung vermittelt wurde: «Das hat man gelernt, dieses Über-Werkzeug und an dem haften viele» (Schulleiter V7). Die Entwicklung hin zum stufenübergreifenden Arbeiten im Rahmen des Werkstattunterrichts erfolgte vor allem durch «das Beobachten der Gruppe». Er erlebt, dass die Fähigkeiten der SchülerInnen in den unterschiedlichen Altersgruppen sehr unterschiedlich sind und nicht immer mit dem Alter zusammenhängen. Dies ermöglicht aus seiner Sicht die Kooperation von SchülerInnen über die verschiedenen Schulstufen hinweg. Auch das gemeinsame Interesse an einem Thema kann SchülerInnen verschiedener Schulstufen für eine Zusammenarbeit motivieren. Er beobachtet, dass es oft Freundschaften zwischen jüngeren und älteren SchülerInnen sind, die eine Zusammenarbeit begründen. Vom Denken in Jahrgangsstufen wegzukommen war auch möglich, weil der Schulleiter «die Angst verloren» hat, seine SchülerInnen zu überfordern. Auf die Frage nach den Inspirationsquellen für den Werkstattunterricht weist der Schulleiter neben den eigenen Beobachtungen auch auf eine Kollegin und die theoretische Auseinandersetzung hin:

Das ist so eine Mischung: zum Teil auch Eigenbeobachtung, eine wunderbare Lehrerin, die diese Struktur angewendet hat, (...) da habe ich als Leiter eine Stunde beobachtet und ich habe das nachher abgekupfert. (lacht) (...) Freinet ist schon der Überbau (...) da habe ich viel Literatur gelesen. (Schulleiter V7)

Am Beispiel der Entwicklung des Werkstattunterrichts wird deutlich, wie komplex die didaktische Umsetzung eines altersgemischten Unterrichts ist, in der die Altersheterogenität als Chance gesehen und genützt wird. Es zeigt sich hier, dass langjährige Erfahrungen mit entsprechender reflexiver Auseinandersetzung notwendig waren, um einen anspruchsvollen altersgemischten Unterricht gestalten zu können.

\section{Umsetzung des altersgemischten Unterrichts in den einzelnen Fächern}

Die Organisation altersgemischter Lernsituationen erleben Lehrpersonen in manchen Fächern als leichter realisierbar als in anderen:

Es ist ganz unterschiedlich von Fach zu Fach. Es ist nicht in jedem Fach gleich einfach und es ist nicht in jedem Fach gleich realisierbar. (Schulleiter SG2)

Vielen Lehrpersonen fällt es relativ leicht, Werken, Bewegung und Sport, Musik oder Bildnerische Erziehung sowie Sachunterricht (Österreich) bzw. «Mensch und Umwelt» (Schweiz) altersgemischt zu organisieren:

In Sachunterricht passiert es doch öfters, dass man dasselbe Thema hat und dann macht man es einfach übergreifend (...) natürlich bekommen dann die Grösseren, sprich die vierte Schulstufe, Zusatzaufgaben. (Schulleiter V1) 
In Deutsch werden die Umsetzungsmöglichkeiten des altersgemischten Unterrichts sehr unterschiedlich erlebt. Grammatikunterricht altersgemischt zu organisieren wird als eher schwierig eingestuft, gemeinsames Lesen hingegen wird häufig praktiziert:

Ich empfinde es auch als sehr positiv, wie sie einander vorlesen, (...) indem sie anderen vorlesen, lernen sie auch, üben sie das Lesen (...) Es ist mit Sicherheit motivierender, jemandem eine Geschichte vorzulesen oder jemandem meinen Aufsatz vorzulesen. (Schulleiterin V8)

Es fällt auf, dass insbesondere im Fremdsprachenunterricht und im Fach Mathematik grosse Unsicherheiten darüber bestehen, wie eine passende Unterrichtskonzeption für altersgemischte Lerngruppen aussehen könnte:

Deutsch und Mensch und Umwelt sind einfach. Was ganz schwierig ist, ist Mathematik, darum haben wir sie dort geteilt. (Schulleiter SG2)

Für die Organisation eines zeitgemässen altersgemischten Unterrichts spielen entsprechende Lernmaterialien eine grosse Rolle. Die meisten der interviewten LehrerInnen zeigen sich mit den in ihren Schulen vorhandenen didaktischen Materialien zufrieden. Der Einsatz des Computers mit individuellen Lernprogrammen stellt für viele Lehrpersonen kleiner Schulen ein wichtiges Element dar, ihren altersgemischten Unterricht öffnen zu können.

\section{Ausblicke}

Im Rahmen dieses Beitrags wurden die Besonderheiten kleiner Schulen im ländlichen Raum, die Chancen und Herausforderungen und die Praxis des altersgemischten Unterrichts beschrieben. Es wurde deutlich, dass der altersgemischte Unterricht in den Schulen sehr unterschiedlich umgesetzt wird. In einzelnen kleinen Schulen wurden sehr innovative Unterrichtsmodelle entwickelt. Hier zeigte sich, dass der Aufbau eines unterrichtsorganisatorisch, pädagogisch und didaktisch durchdachten Konzepts eine anspruchsvolle Aufgabe darstellt. Lehrpersonen kleiner Schulen können aufgrund ihrer langjährigen Erfahrungen im Umgang mit altersheterogenen Lerngruppen und ihrem Wissen über die Umsetzungsmöglichkeiten altersgemischten Unterrichts viel zur Weiterentwicklung der Grundschule beitragen. Diese langjährigen Erfahrungen sollten jedoch verstärkt in den schulpädagogischen Diskurs einfliessen, um das Transferpotential für aktuelle Reformen zu nutzen. Eine verstärkte empirische Auseinandersetzung mit kleinen Schulen würde ermöglichen, Vorurteile - negative und positive - kritisch zu hinterfragen. Negative Vorurteile bestehen insofern, dass kleine Schulen im ländlichen Raum zum Teil immer noch als rückständig und der hier praktizierte altersgemischte Unterricht oft als blosser «Notnagel» gesehen wird. Wie gezeigt werden konnte, sind sie zum Teil viel mehr als das. Mit positiven Vorurteilen sind hier vor allem jene gemeint, die eine unkritische Beschreibung ländlicher Lebenswelten als Idyll und Dorfschulen als Lernorte 
beschreiben, «in dem noch alles in Ordnung ist». Mit der Darstellung der Herausforderungen und der unterschiedlichen Kontextfaktoren kleiner Schulen können Idealisierungstendenzen entgegengewirkt werden. Weder eine Nichtbe $\neg$ achtung bzw. Abwertung noch eine Idealisierung helfen zu verstehen, worin die Chancen aber auch die Herausforderungen kleiner Schulen und des hier praktizierten altersgemischten Unterrichts liegen.

\section{Literaturverzeichnis}

Amt der Vorarlberger Landesregierung. (2011). Schulstatistik 2009/10. Bregenz: Landesstelle für Statistik.

Arnold, M. L., Newman, J. H., Gaddy, B. B. \& Dean, C. B. (2005). A look at the condition of rural education research: Setting a direction for future research. Journal of Research in Rural Education, 20 (6), 1-25.

Berthold, B. (2008). Unterricht entwickeln in der Schuleingangsphase: Grundlagen, Ziele, Anregungen. Baltmannsweiler: Schneider-Verlag Hohengehren.

Breuer, S. \& Simon, R. (2001). Jahrgangsübergreifender Unterricht - ein notwendiger Reformansatz. Die Grundschulzeitschrift, 150, 32-35.

De Boer, H., Burk, K. \& Heinzel, F. (Hrsg.). (2007). Lehren und Lernen in jahrgangsgemischten Klassen. Frankfurt am Main: Grundschulverband - Arbeitskreis Grundschule.

Feuser, G. (2002). Momente entwicklungslogischer Didaktik einer Allgemeinen (integrativen) Pädagogik. In H. Eberwein \& S. Knauer (Hrsg.), Integrationspädagogik. Kinder mit und ohne Beeinträchtigung lernen gemeinsam (6. Aufl.) (S. 280-294). Basel: Beltz Verlag.

Fickermann, D., Weishaupt, H. \& Zedler, P. (Hrsg.). (1998). Kleine Grundschulen in Europa: Berichte aus elf europäischen Ländern. Weinheim: Deutscher Studien-Verlag.

Grabher, N. (2002). Jahrgangsmischung an einer Kleinschule: Über das Müssen und das Wollen der Altersmischung. Erziehung und Unterricht, 152 (3-4), 511-514.

Gutiérrez, R. \& Slavin, R. E. (1992). Achievement effects of nongraded elementary school: A best evidence synthesis. Review of Educational Research, 62, 333-376.

Hagstedt, H. (2003). Lernen durch Lehren - zwischen Reformanstrengungen und Forschungsbedenken. In R. Laging (Hrsg.), Altersgemischtes Lernen in der Schule (S. 30-38). Baltmannsweiler: Schneider-Verlag Hohengehren.

Hahn, H. \& Berthold, B. (Hrsg.). (2010). Altersmischung als Lernressource. Impulse aus Fachdidaktik und Grundschulpädagogik. Baltmannsweiler: Schneider-Verlag Hohengehren.

Hanke, P. (2007). Jahrgangsübergreifender Unterricht in der Grundschule. Konzepte, Befunde und Forschungsperspektiven. In H. de Boer, K. Burk \& F. Heinzel (Hrsg.), Lehren und Lernen in jahrgangsgemischten Klassen (S. 309-324). Frankfurt am Main: Grundschulverband - Arbeitskreis Grundschule.

Hargreaves, L., Kvalsund, R. \& Galton, M. (2009). Reviews of research in rural schools and their communities in British and Nordic countries: Analytical perspectives and cultural meaning. International Journal of Educational Research, 48, 80-88.

Heinzel, F. (2007). Altersstufen, Altersmischung und Generationenbeziehungen in der Grundschule. In H. de Boer, K. Burk \& F. Heinzel (Hrsg.), Lehren und Lernen in jahrgangsgemischten Klassen (S. 32-43). Frankfurt am Main: Grundschulverband Arbeitskreis Grundschule.

Kalaoja, E. \& Pietarinen, J. (2009). Small rural primary schools in Finland: A pedagogically valuable part of school network. International Journal of Educational Research, 48, 109-116.

Krappmann, L. \& Oswald, H. (1995). Alltag der Schulkinder: Beobachtungen und Analysen von Interaktionen und Sozialbeziehungen. München: Juventa.

Kucharz, D. (2009). Jahrgangsunterricht und jahrgangsübergreifender Unterricht. In S. 
Blömeke, T. Bohl, L. Haag, G. Lang-Woitasik \& W. Sacher (Hrsg.), Handbuch Schule. Theorie - Organisation - Entwicklung (S. 339-343). Bad Heilbrunn: Klinkhardt.

Kucharz, D. \& Wagener, M. (2009). Jahrgangsübergreifendes Lernen: Eine empirische Studie zu Lernen, Leistung und Interaktion von Kindern in der Schuleingangsphase (3. Aufl.). Baltmannsweiler: Schneider-Verlag Hohengehren.

Laging, R. (Hrsg.). (2003). Altersgemischtes Lernen in der Schule (2., korrigierte Aufl.). Baltmannsweiler: Schneider-Verlag Hohengehren.

Müller, R., Keller, A., Kerle, U., Raggl, A. \& Steiner, E. (2011). Schule im alpinen Raum. Reihe FokusBildungSchule (Bd. 2). Innsbruck: Studienverlag.

Oelkers, J. (1989). Reformpädagogik. Eine kritische Dogmengeschichte. Weinheim: Juventa.

Peschel, F. (2007). Vom Abteilungsunterricht zum offenen Unterricht. In H. de Boer, K. Burk \& F. Heinzel (Hrsg.), Lehren und Lernen in jahrgangsgemischten Klassen (S. 104-114). Frankfurt am Main: Grundschulverband - Arbeitskreis Grundschule.

Poglia, E. \& Strittmatter, A. (1983). Die Situation der Mehrklassenschulen in der Schweiz. Ergebnisse einer Untersuchung durchgeführt im Rahmen des Nationalfonds-Projekts "Regionale Verschiedenheiten im Bildungswesen. Genf: Schweizerische Konferenz der kantonalen Erziehungsdirektoren.

Raggl, A. (2011). Altersgemischter Unterricht in kleinen Schulen im alpinen Raum. In R. Müller, A. Keller, U. Kerle, A. Raggl \& E. Steiner, Schule im alpinen Raum (S. 231-305). Reihe FokusBildungSchule (Bd. 2). Innsbruck: Studienverlag.

Rossbach, H.-G. (2003). Empirische Vergleichsuntersuchungen zu den Auswirkungen von jahrgangsheterogenen und jahrgangshomogenen Klassen. In R. Laging (Hrsg.), Altersgemischtes Lernen in der Schule (S. 80-91). Baltmannsweiler: Schneider-Verlag Hohengehren.

Rückriem, G. M. (1965). Die Situation der Volkschule auf dem Lande. Soziologische Studien und pädagogische Überlegungen. München: Kösel.

Sandfuchs, U. (1997). Grosse oder kleine Schulen? Anmerkungen zum Streit um die optimale Schulgrösse. In U. Sandfuchs, E.-M. Stange \& S. Kost (Hrsg.), Kleine Grundschule und jahrgangsübergreifendes Lernen. Schülerrückgang als pädagogische Herausforderung (S. 1-20). Bad Heilbrunn: Klinkhardt.

Sandfuchs, U., Stange, E.-M. \& Kost, S. (Hrsg.). Kleine Grundschule und jahrgangsübergreifendes Lernen. Schülerrückgang als pädagogische Herausforderung. Bad Heilbrunn: Klinkhardt.

Starr, K. \& White, S. (2008). The small rural school principalship: Key challenges and crossschool responses. Journal of Research in Rural Education, 23 (5), 1-12.

Trachsler, E. (2008). Altersdurchmischte Klassen in der Thurgauer Volksschule. Eine explorative Studie zu einer vielversprechenden Schulform zwischen Tradition und Innovation. Kreuzlingen: Pädagogische Hochschule Thurgau.

Tillmann, K.-J. (2004). System jagt Fiktion: Die homogene Lerngruppe. Friedrich Jahresheft, 12, 6-9.

Waugh, D. (1990). Implementing change in the small primary school. Aspects of Education, 43, $1-13$.

Vulliamy, G. \& Webb, R. (1995). The implementation of the National Curriculum in small primary schools. Educational Review, 47 (1), 25-41.

Wagener, M. (2007). Gegenseitiges Helfen im altersgemischten Unterricht. In H. de Boer, K. Burk \& F. Heinzel (Hrsg.), Leben und Lernen in jahrgangsgemischten Klassen (S. 124-133). Frankfurt am Main: Grundschulverband - Arbeitskreis Grundschule.

Schlagworte: Altersgemischter Unterricht, kleine Schulen, ländlicher Raum, Grundschulen 


\section{L'enseignement dans les classes à degrés multiples dans les zones rurales}

\section{Résumé}

Cet article décrit les caractéristiques particulières des "petites écoles» ainsi que les pratiques de l'enseignement dans les classes à degrés multiples de différentes régions rurales des Alpes. Dans ces régions, l'enseignement dans les classes à degrés multiples est avant tout une mesure dictée par la nécessité de garder ouvertes des écoles dont le nombre d'élèves est peu élevé. Malgré des difficultés organisationnelles reconnues, les directrices et directeurs d'école ainsi que les enseignantes et enseignants qui ont participé au projet de recherche «Les écoles des régions alpines» valorisent le potentiel pédagogique de l'enseignement dans les classes à degrés multiples. Ainsi, certains profitent la marge de manœuvre que permet cette organisation pour développer des modèles didactiques innovants. Il s'avérait que la mise en ouvre de l'enseignement dans les classes à degrés multiples soit un défi stimulant pour le personnel enseignant, en particulier dans les «très petites écoles» qui n'ont qu'un-e seul-e enseignant-e.

Mot-clés: L'enseignement dans les classes à degrés multiples, petites écoles, régions rurales, école élémentaire

\section{L'insegnamento in "classi di età mista" in piccole scuole dello spazio rurale}

\section{Riassunto}

L'articolo seguente presenta le particolarità delle piccole scuole e la prassi dell'insegnamento in "classi di età mista" di piccole scuole nelle varie regioni dello spazio alpino rurale. L'insegnamento in "classi di età mista" nelle regioni rurali rappresenta in primo luogo una misura organizzativa necessaria per mantenere piccole scuole con un numero esiguo di scolari. Sia le direzioni scolastiche che il corpo insegnanti delle scuole che hanno partecipato al progetto «La scuola nello spazio alpino" hanno individuato nella forma ridotta dell'organizzazione scolastica e nell'insegnamento in classi di età mista anche una valida opportunità pedagogica; alcune scuole sfruttano addirittura tali contingenze per sviluppare modelli didattici innovativi. Si è visto inoltre come l'applicazione dell'insegnamento in "classi di età mista" si rivela non priva di ostacoli, soprattutto nelle scuole molto piccole con un insegnante unico.

Parole chiave: Insegnamento in classi di età mista, piccole scuole, regioni rurali, scuola elementare 


\section{Teaching in Mixed Aged Classes in Small Rural Schools}

\section{Summary}

The characteristics of «small schools» are described in this paper together with the teaching and learning practices in mixed aged classes of rural-alpine schools. In these rural areas, mixed age classes are mainly an organizational measure to keep schools with a low number of pupils open. However, teachers and headteachers involved in the research project "Schools in alpine regions" also celebrate the pedagogical advantages of small schools and mixed aged learning. Some of them take advantage of their autonomy to develop innovative teaching and learning practices. Yet, the research also shows that mixed age classes involve important challenges for teachers, especially for those in very small 'one-teacher-schools'.

Key words: Mixed aged classes, small schools, rural regions, elementary schools 
